\title{
HYPOTHESIS
}

\section{DNA bioelectric field: a futuristic bioelectric marker of cancer, aging and death - A working hypothesis}

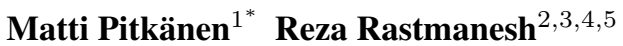 \\ ${ }^{1}$ Independent Physics Researcher, Rinnekatu 2-4 8A, Karkkila 03620, Finland \\ ${ }^{2}$ Member of The Nutrition Society, London, UK \\ ${ }^{3}$ Member of The American Physical Society, USA \\ ${ }^{4}$ Independent Nutrition and Biology Researcher, Tehran, Iran \\ ${ }^{5}$ Member of Society for Conservation Biology, USA
}

\section{Check for updates}

*Correspondence to: Matti Pitkänen, Independent Physics Researcher, Rinnekatu 2-4 8A, Karkkila 0362 0 , Finland;

E-mail: matpitka6@gmial.com

Received: December 16, 2020;

Accepted: February 19, 2021;

Published: March 3, 2020

Citation: Pitkänen M and Rastmanesh R. DNA bioelectric field: a futuristic bioelectric marker of cancer, aging and death $-\mathrm{A}$ working hypothesis. Curr Cancer Rep, 2021, 3(1): 68-80

https://doi.org/10.25082/CCR.2021.01.002

Copyright: () 2021 Matti Pitkänen, et al. This is an open access article distributed under the terms of the Creative Commons Attribution License, which permits unrestricted use, distribution, and reproduction in any medium, provided the original author and source are credited.

\section{cc) (7) \&}

\section{Editor's Note}

Bio-electrical phenomena were first discovered by Luigi Galvani in the 18th century. With the development of molecular and systems biology research in the last five decades, nowadays the comprehensive understanding of cell behaviors based on a combination of genetics, physics and physiology seems ready to come out at one's call on a bioelectrical conceptualization of cells, especially, recent reports demonstrated that externally applied electrical fields can modulate multicellular processes such as regeneration in plant and vertebrate tissue. All these provide not only plausible explanations for many cell behaviors, but also a new framework to re-formulate much of the existing knowledge in cell physiology, i.e. electrical and chemical potential differences across cellular membranes, or electrochemical gradients (ion motive forces, IMFs) and membrane potential (MP). Interestingly, increasing findings at molecular levels directly lead to the recent proposition that multicellular organization and development more broadly, can, and should, be studied as a bioelectrical paradigm. This framework offers a new synthesis bridging molecular studies with a bioelectrical basis of cellular physiology. The resulting science can have a transformative effect on our understanding of cellular behaviors and pave a novel way to its direct control through predictive bioelectrical marking and/or engineering. Briefly, bioelectricity has become a holistic approach to understand diverse cell behaviors, even sub-cellular events. Telomeres, a specialized DNA-protein structure with a repetitive sequence of DNA at the ends of eukaryotic chromosome, play important role in keeping chromosome intact and genome stability. DNA end replication creates a $3^{\prime}$ single-stranded overhang at each end of the linear chromosome. The $3^{\prime}$ overhang fold back and invade into the dsDNA region of telomere to form loop structure avoiding potential damage on DNA. Usually, telomere length gets shortened after each round of replication, till becomes critically short and unable to form the loop. So the telomere integrity depends on telomere length maintenance and genome stability, the so-called telomere homeostasis. Recently, telomere homeostasis has gained more attention, because its persistent imbalance will result in a spectrum of disorders -telomeropathies, such as dyskeratosis congenita (DKC), aplastic anemia, fanconi anemia and cancer, etc. This background provides an excellent opportunity to put forward novel hypothesis and establish novel predictive, prognostic and therapeutic biomarkers differentially in different cell types or as a combined bioelectric marker for "whole-body" as a derivation of mathematical formulations. The following hypothesis is a good case in point, which suggested DNA's bioelectric field as a novel bioelectric marker for prognostic and diagnostic purposes in researches of cancer, aging, surgery grafts and rejuvenation for the first time. It may open new areas of research on the neglected capacity of telomere and DNA research in diagnosis of a broad array of diseases, physiologic and pathologic conditions. This hypothesis is necessary for designing tailor-made micro-electrochemical impedance spectroscopy technologies, and consequently, pilot studies designed a priori are needed to test the hypothesis. CCR editorial office looks forward to further active exploration of relevant aspects from scientists in basic and clinic research all over the world. 
Abstract: Telomeres are associated with the ends of DNA double strands. The lengths of the telomeres are controlled by the telomerase enzyme. The shortening of the telomeres is known to relate to aging. In cancers, telomere lengths are abnormally short. Telomeres could act as buffers shielding the part of DNA coding for the proteins. For cancer cells, germ cells and stem cells, the length of the telomeres is not varying. There is an analogy with microtubules, which are highly dynamical and carry a longitudinal electric field, whose strength correlates with the microtubule length. Could sticky ends generate a longitudinal field along DNA double strand with strength determined by the lengths of the sticky ends? In the standard picture, the flux of the longitudinal electric field would be proportional to the difference of the negative charges associated with the sticky ends. In Topological Geometrodynamics (TGD) framework, DNA strands are accompanied by the dark analog of DNA with codons realized as 3-proton units at magnetic flux tubes parallel to DNA strands and neutralizing the negative charge of ordinary DNA except at the sticky ends. This allows considering the possibility that opposite sticky ends carry opposite charges generating a longitudinal electric field along the magnetic flux tube associated with the system. DNA/Telomere bioelectric field could serve as a novel bioelectric marker to be used for prognostic and diagnostic purposes in researches of cancer, aging, surgery grafts and rejuvenation. We propsed that DNA bioelectric field can be used as a futuristic bioelectric marker of cancer, aging and death.

Keywords: bioelectric marker, cancer, aging, rejuvenation, telomere, early diagnosis

\section{Introduction}

The motivation for this article was the question whether there could be a longitudinal electric field associated with DNA and whether the reduction of its strength could serve as a bioelectric marker of cancer, aging and death. This could be the case if the length of DNA correlates with the strength of this electric field. The natural question is whether the length of the negatively charged sticky end of DNA could determine the strength of this electric field.

\subsection{Could DNA be a ferroelectret and could the length of sticky ends control the strength of the longitudinal electric field?}

DNAs and RNAs have bioelectrets [1,2] but the question whether they are bioferroelectrets possessing a constant longitudinal electric field in the absence of external electric field is an open question.

Telomeres are associated with the ends of DNA double strands. The lengths of telomeres are controlled by the telomerase enzyme. The shortening of telomeres is known to relate to aging. For cancer cells, germ cells and stem cells the length of the telomeres is not varying. In cancer their lengths are abnormally short. Telomeres could act as buffers shielding the part of DNA coding for proteins. Telomeres have "sticky ends" assignable only to the second DNA strand and carrying negative charge. What their function could be? Could telomere lengths correlate with the lengths of the sticky ends and what could control their lengths?

There is an analogy with microtubules, which are highly dynamical and carry a longitudinal electric field, whose strength correlates with the microtubule length. Could the sticky ends generate a longitudinal field along DNA double strand with strength determined by the lengths of the sticky ends? In the standard picture the flux of the longitudinal electric field would be proportional to the difference of the negative charges associated with the sticky ends.

In a conceptual framework based on Topological Geometrodynamics (TGD) [3,4], which is a proposal for a unification of fundamental interactions inspiring a vision about consciousness and quantum biology, DNA strands are accompanied by the dark analog of DNA with codons realized as 3-proton units neutralizing the negative charge of ordinary DNA except at sticky ends. If the dark double strand accompanies also the sticky end, the total charge is positive. If not, it is negative. This allows to consider the possibility that opposite sticky ends have opposite charges so that there is a long dipole like entity carrying longitudinal electric flux proportional to the common length of sticky ends.

\subsubsection{Experimental signatures}

Also in standard physics based picture (no dark DNA), an external electric field created by the polarization of the nucleotides A, T, C, G in an external electric field is possible [2] This would mean electrect property, not yet ferroelectricity. The model for the phenomenon suggests that ferro-electricity could result in the sense that the polarization is non-vanishing also 
in absence of the external electric field so that the nucleotide rather than entire DNA strand would be an electric analog of ferromagnet.

In this case the behavior in the external electric field is different from that for fer-roelectric DNA double strand: DNA double strand itself would not experience a direct torque in the external electric field. The effective polarization per nucleotide predicted by TGD is at least by a factor 2.5-7.5 stronger than standard model polarizations so that the model can be tested. Furthermore, ferroelectricity of DNA in TGD sense requires DNA double strands and would be present for single DNA strand.

The secod testable prediction is the possibility of currents running along DNA double strand in the longitudinal electric field even without external electric field. External field would however add to the ferroelectric field of oriented DNA double strands and lead to an anomalously high conductivity. Another test would be based on the ferroelectret property of living tissues, which could be caused both by DNA and protein ferro-electricity. Living tissues are indeed known that be ferroelectres as the phenomena of pyroelectricity, piezoelectrity -studied first by [1] and the polarization in an external electric field demonstrate. Ahlenstaedt proposed that the permanent dipole like character (ferroelectricity) of the linear biomolecules gives rise to their bioferroelectricity.

\subsubsection{Connection with consciousness}

An analogy with the findings of Becker [5] about the electric fields along the body axis emerges. Becker found that the direction of this field determines whether the organism is awake or in a sleep state. The weakening of these fields leads to aloss of consciousness. TGD inspired theory of consciousness predicts that even sysems like DNA can be conscious and the fractality of TGD Universe suggests that the physical correlates of consciousness are same in all scales.

Could the direction and strength of the electric field of DNA correlate with consciousness at this level? In TGD based quantum measurement theory extending to a theory of consciousness the arrow of time changes in "big" state function reductions (BSFRs), which mean "death" and "reincarnation" with opposite arrow of time [3]. By the tensorial properies of electromagnetic field tensor the arrow of time correlates also with the direction of the electric field. This leads to ask whether the change of the arrow of time in BSFR could change the direction of the DNA bioelectric field, and one ends up with a simple mechanism for this based on the analog of Becker's DC currents along DNA as proton currents.

\subsection{Telomere length, cancer, and DNA ferroelectricity}

Compelling evidence suggest that there is an inverse relationship between telomere length and both different types of cancer incidence and mortality [6-8] suggesting that the control of telomere length by telomerase enzyme is impaired $[9,10]$.

Almost in all cancer cells, telomere length is shortened [11,12]. Telomere shortening accompanies ageing [13]. Even in stem cells, except for embryonic stem cells and cancer stem cells, there are overwhelming evidence that telomere shortening occurs during replicative ageing, though at a lower rate than that in normal somatic cells [14]

In this picture, the simplest possibility is that telomeres act as buffers, and the strength of the longitudinal electric field controlled by the length of sticky ends controls the length of telomeres and thus of DNA. Sticky ends would be the key control knobs used by telomerase enzyme, and magnetic body (MB) of the system would be the ultimate controller.

Apart from some exceptions, telomere length in DNA is shortened in almost all cell types during aging and some diseases, based on the level of telomerase activity or its absence [8]. Ageing could be purposefully induced since eternal life would be a metabolic catastrophe from the perspective of population overgrowth and evolution [15].

This motivated us to propose the hypothesis that DNA bioelectricity changes over time and depends on disease progression and severity. This provides an excellent opportunity to establish novel predictive, prognostic and therapeutic biomarkers differentially in different cell types or as a combined bioelectric marker for "whole-body" as a derivation of mathematical formulations. This hypothesis is necessary for designing tailor-made microelectrochemical impedance spectroscopy technologies, and consequently, pilot studies designed a priori are needed to test our hypothesis. Along with replication of well-designed pilot study with a more diverse population and larger sample size, it will be needed to address questions about cut-offs or personalized normal ranges for differential and mean whole-body DNA's bioelectric field in a longitudinal study in a prospective manner. With this brief background, the aim of this paper is to suggest DNA bioelectric field as a novel bioelectric marker to be used for prognostic and 
diagnostic purposes in researches of cancer, aging, surgery grafts and rejuvenation, for the first time.

\section{Could the sticky ends make DNA double strand a ferro-electret?}

In the sequel the idea that sticky ends make the DNA double strand + its dark counterpart a ferro-electret carrying longitudinal electric field is considered. The longitudinal electric field is non-vanishing also in standard framework without dark DNA if the lengths at the ends of the DNA double strand are different. This field would be analogous to the electric field along the body axis. As Becker found [5], its direction determines whether the system is in wake-up state or sleep and the same could be true also for DNA+ dark DNA system.

\subsection{Different ends of DNA double strand}

There are Wikipedia articles about telomeres [16] and about the so called overhangs/sticky ends at their ends [17]. The video about telomeres and sticky ends [18] is very helpful for a non-specialist.

For readers' convenience is appropriate to summarize first the basic facts about telomeres and sticky ends. There is a variety of different ends of DNA double strand and of telomere.

(1) Blunt ends contain two paired bases so that they do not define a full codon.

$$
\begin{aligned}
& 5^{\prime} \text { - CTGATCTGACTGATGCGTATGCTAGT - 3' } \\
& 3^{\prime} \text { - GACTAGACTGACTACGCATACGATCA - 5' }
\end{aligned}
$$

Straight cut by exonuclease enzyme produce blunt ends.

(2) Overhangs are short, minimally just one nucleotide A in 3' end: one could have for instance following configuration

$$
\begin{aligned}
& 5^{\prime} \text { - ATCTGACTA - 3' } \\
& 3^{\prime} \text { - TAGACTGA - 5' }
\end{aligned}
$$

Overhangs are most often palindromic.

(3) An example of longer sticky end is following:

$$
5^{\prime} \text { - ATCTGACT } 3^{J} \text { - TAGACTGACTACG }
$$

The length of the unpaired portion of sticky end can be hundreds of nucleotides.

(4) Frayed ends correspond to sequence of basic pairs breaking the A-T, C-G pairing rules.

$$
\begin{aligned}
& 5^{\prime} \text { - ATCTGACTAGGCA - } 3^{\prime} \\
& 3^{\prime} \text { - TAGACTGACTACG - } 5^{\prime}
\end{aligned}
$$

\subsection{Empirical evidence for the ferroelectret property of DNA}

To the best of our knowledge, there is no reported evidence for longitudinal static electric fields in DNA in an extensive Web search. This might be simply because of inability to measure them in the past. Indeed, a model for DNA nucleotides A,T,C,G as ferroelectrets based solely on [19] and would imply that also DNA can be ferroelectret. This could in a special case give rise to a longitudinal electric field, and if there is an electric field in the absence of external electric field (spontaneous ferroelectricity), it could be also in the direction of DNA strand.

The reported existence of electric currents along DNA perhaps analogous to Becker's DC currents is one indirect evidence for the longitudinal electric field. A very interesting test would be so called DNA crystals [20]. See also the popular article [21] in electric field, heated, or put under mechanical stress.

DNA is analogous like cell interior being negatively charged with one negative charge per nucleotide assignable to the phosphate. The stability of DNA against Coulomb force is however not well-understood and TGD would solve the problem with a pairing of DNA strand with a parallel helical flux tube carrying 3 dark protons per codon with dark proton triplet realizing genetic codon. Ordinary chemical codons would be a secondary representation of the code. Could this make possible ferroelectret property of DNA?

\subsection{Could the sticky ends of the telomeres give rise to a longitudi- nal electric field along DNA?}

In the standard picture about DNA, different negative charges at the sticky ends could give a longitudinal electric field proportional to the difference of the charges. DNA double strand would however have a net charge now. Second possibility is that the nucleotides behave as 
dipoles even in the absence of the external electric field. If these dipoles are forced to be parallel to DNA by an external electric field they give rise to a longitudinal electric field.

TGD based view is that DNA is paired with dark analog of DNA. This view leads to the suggestion that sticky ends/overhangs give rise to positive or negative charges at the end of DNA and that opposites at the ends of DNA generate strong longitudinal electric field along DNA. For DNA with blunt ends there would be no electric field.

What would be needed for chromosome as dipole like entity is that the ends of the chromosome carrying the telomeres have charges of opposite sign: in the simplest case they would have the same magnitude so that one would have a dipole.

\subsubsection{Could telomeres be analogous to microtubules?}

Microtubules are highly dynamical having a varying length. They also have a longitudinal electric field [22,23]. Likewise, the the ends of chromosomes are dynamical and their length is changing and controlled by the telomerase enzyme [11,24]. Could telomeres or entire chromosomes be analogous to microtubules? Could chromosomes [25] carry longitudinal electric fields? That would not be surprising since living matter is populated by ferroelectrets [26].

Remark: The option that only telomeres could carry these fields would require that the joint between the coding portion of DNA and telomere is charged. This does not look natural.

Due to the properties of the electric field under time reversal, the direction of the bioelectric field would in TGD Universe correlate with the arrow of time [3,26] changing in "big" (ordinary) state function reductions (BSFRs) meaning "death" or "falling asleep" and reincarnation with an opposite arrow of time. In particular, sleep could correspond to conscious experience but with a different arrow of time at some level of the hierarchy of layers of MB [27] serving as master controlling the biological body (BB).

Remark: The hierarchy of Planck constants $h_{e f f}=n h_{0}$ labelling phases of ordinary matter behaving like dark matter predicts $[28,29]$ macroscopic quantum coherence explaining the coherence of biomatter. This allow BSFRs in arbitrarily long length and time scales, for instance, the scales of chromosomes.

The first guess motivated by the findings of Becker about bioelectric fields [5] is that when the telomere shortens, the electric field associated with DNA weakens, and eventually the organism dies [13] . Telomere length is controlled by telomerase enzyme and for stem cells, germ cells and cancer cells the shortening does not occur.

Telomeres are dynamical and could somehow provide DNA with a longitudinal electric field closely related to this dynamics. The strength of the electric field associated with the DNA double strand could correlate with the properties of telomeres and in particlar, with the lengths of their negatively charged sticky ends at the ends of the chromosome.

\subsubsection{The TGD based model for DNA as ferroelectret}

Although most of the telomere has a normal base-pairing, there is an additional unpaired nucleotide sequence - overhang - associated with either strand. In the minimal case, it is just one nucleotide A. What could this mean in TGD framework: could it give the desired constant electric field along DNA strand? Is its strength proportional to the length of the overhang determined by the number of its nucleotides? There would be 1 negative charge per nucleotide

(1) Suppose that both strands are accompanied by dark DNA strands parallel to them and having opposite charge neutralizing the DNA in the scale of this pairing. Dark codon would be identified as a 3-proton unit. Dark RNA, tRNA and amino-acids are predicted. Vertebrate genetic code is predicted correctly in the sense that the number of DNA codons corresponding to given dark amino-acid is the same as for vertebrate genetic code $[29,30]$.

(2) What could be the counterpart of the sticky end for dark DNA sequence? Suppose that the dark DNA strands be equally long so that there would be no symmetry breaking. This leaves two natural options for a given sticky end.

(a) Both dark DNA strands have portions associated with the sticky end. Since the sticky end/overhang would be neutralized, this would give for the end of the double strand a positive charge $Q=n e, n$ is the number of nucleotides in the sticky end.

(b) Both dark DNA strand portions are missing at the sticky end. Now the charge would be negative and equal to the charge $Q=-n e$ of the sticky end.

(3) The magnitude of the electric field along DNA flux tube created by a single sticky end would be

$$
E=\frac{Q}{S}=\frac{n e}{S}
$$


where $\mathrm{S}$ is the thickness of the system DNA + dark DNA. The fields of the sticky ends sum up and there would be a net electric field along DNA double strand +dark DNA given by

$$
E=\frac{Q_{1}-Q_{2}}{S}=\frac{e\left(n_{1}-n\right)}{S}
$$

One can consider two options:

Option I: There is dark DNA present (TGD option) and the situation is a) at the first end of the chromosome and b) at the opposite end. One obtains opposite signs of charges $Q 1=n l e$ and $Q 2=-n 2 e$ and electric field is $E=(n 1+n 2) e / S$.

Option II: There is no dark DNA (standard physics option). The charges at the sticky ends are negative and one has $E=e(n 1-n 2) / S$.

(4) The video about telomeres [18] suggests that the sticky ends are associated with different DNA strands and are of the same length. For the standard physics option (no dark DNA) charges at the sticky ends have the same sign and one has $E=e(n 1-n 2) / S$. The field vanishes for Option II and equals to $E=2 n / S$ for Option I. This field would be quite strong. The electric fields at opposite ends of the chromosome sum up and cancel each other along DNA if the charges are of the same sign : there is however positive interaction energy causing a repulsive force. For the TGD option the Coulomb energy is negative. For the standard physics option it would be positive and would not favor the stability of DNA.

\subsubsection{Quantitative estimates}

In the sequel some simple quantitative estimates are performed.

(1) Minimization of electrostatic energy taking into account only the nearest neighbor interactions

The system must minimize its electrostatic energy to be stable. Assume that the charges of the overhangs are opposite: $n_{1} \neq-n_{2}=n$. For the more general situation with $n_{1}=n_{2}$. For the same sign for $n_{1}$ and $n_{2}$ there would be repulsion between the ends of DNA.

(a) In this case overhangs would give a negative contribution to the electrostatic energy of the system.

$$
E_{\text {ends }}=-\frac{\mathrm{n}^{2} \mathrm{e}^{2} \mathrm{~L}}{\mathrm{~S}}
$$

where $\mathrm{L}$ is the length of DNA double strand without overhangs and $\mathrm{S}$ is it transversal area. Otherwise the contribution is positive.

(b) The negative electrostatic energies between dark strand and ordinary strand with opposite charges. There are two pairs of this kind. In the first approximation one has:

$$
E_{\mathrm{OD}}=-2 N \frac{e^{2}}{\mathrm{R}_{\mathrm{OD}}}
$$

$N$ is the total number of nucleotides in DNA without overhangs and $R_{O D}$ is the distance between dark and ordinary DNA strands. One has $N=(d n / d l) L$, where $d n / d l$ is the number of codons per unit length. One has approximately $d n / d l=10$ nucleotides per nanometer.

This gives:

$$
E_{O D}=-2 \frac{(d n / d l) e^{2} L}{R_{O D}}
$$

The ratio of the two negative contributions tending to stabilize the system is:

$$
r=\frac{E_{O D}}{E_{\text {ends }}}=2 \frac{\left(\frac{d n}{d l}\right) S}{R_{O D}} \cong \frac{20 S}{n m} \times \mathrm{R}_{\mathrm{OD}}
$$

(c) There are positive electrostatic interaction energies between dark strands with distance $R$ $=R_{D D}$ and ordinary strands with distance $R=R_{O O}$. The energy is given by:

$$
E=\frac{N e^{2}}{R}=\frac{\left(\frac{d n}{d l}\right) e^{2} L}{R}
$$

The total contribution to the electrostatic energy is positive and given by:

$$
E_{O O}+E_{D D}=(d n / d l) e^{2} L \times\left(\frac{1}{R_{O O}}+\frac{1}{R_{D D}}\right)
$$

The total electrostatic energy in this approximation is:

$$
E=e^{2} L-\frac{n^{2}}{S}-2\left(\frac{d n}{d l}\right)\left(\frac{1}{R_{O D}}-\frac{1}{R_{O O}}-\frac{1}{R_{D D}}\right)
$$


(d) The generalized electrostatic force in the longitudinal direction is given by:

$$
F=-\frac{d E}{d L}=-e^{2}\left[\frac{n^{2}}{S}-2\left(\frac{d n}{d l}\right)\left(\frac{1}{R_{O D}}-\frac{1}{R_{O O}}-\frac{1}{R_{D D}}\right)\right]
$$

For $n>n_{\min }$ DNA tends to get longer and for $n<n_{\min }$ it tends to get shorter.

(e) In equilibrium this force must vanish. $F=0$ condition fixes the number $\mathrm{n}$ of nucleotides in the sticky end:

$$
n^{2}=n_{0}^{2}=\left(\frac{d n}{d l}\right) \times S\left[\frac{2}{R_{O D}}+\frac{1}{R_{O O}}+\frac{1}{R_{D D}}\right]
$$

This gives:

$$
n=n_{\min }=\sqrt{\left(\frac{d n}{d l}\right) S / R_{D D}} \times \sqrt{-2 \frac{R_{D D}}{R_{O D}}+\frac{R_{D D}}{R_{O O}}+1}=\sqrt{\frac{10 S}{R_{D D} n m}} \sqrt{-2 \frac{R_{D D}}{R_{O D}}+\frac{R_{D D}}{R_{O O}}+1}
$$

Note that the condition $n_{\min }>0$ requires that without the overhangs at the end, the configuration would be unstable. So

$$
2 \frac{R_{D D}}{R_{O D}} \geq \frac{R_{D D}}{R_{O O}}+1
$$

must hold true. Since the right-hand side is larger than unity one must have $2 R_{D D}>R_{O O}$. As special case one could have a maximally symmetric DODO type configuration with $R_{O O}$ $=R_{D D}=R_{O D}$ for which the above inequality becomes equality and one has $n=0 . n=1$ is realized rather generally and is maximally near to this situation.

(f) $n$ would not depend on the length $L$ of the chromosome in the approximation taking into account only the nearest neighbor interactions between various DNA codons. Taking them into account implies that the electrostatic energy is a nonlinear function of $L$ and $n_{\min }$ is predicted to depend on $L$ - probably the dependence is weak suggesting that the dependence of $L=L$ (coding) $+L$ (telomere $)$ - or actually the telomere length $L$ (telomere) - on $n_{\min }$ is strong so that it would be an ideal control variable.

(g) The increase of the length $n$ of the overhang creates a force increasing the length of DNA and its reduction does the opposite. One can say the situation is critical and that $n=n_{\text {min }}$ stabilizes the situation. The reduction of the length of overhang below critical value would have disastrous effect.

This model is certainly not the only one that one can imagine and involves drastic approximations since only the nearest neighbour Coulomb interactions has been taken into account. Also the sticky ends of the chromosome could have different lengths and thus charges so that the chromosome would have a net charge and the stable length for DNA would depend on this charge.

Also the distances between various DNA strands serve as parameters and the stable length depends on these parameters: these parameters could depend on chemical param- eters like $\mathrm{pH}$ and thermo-dynamical parameters. The length of the sticky end is expected to vary also during the life span of the chromosome and also depend on how many DNA replications preceded the generation of the chromosome. The length of the sticky end has spectrum and implies a spectrum for the telomere length since the length $L$ (coding) of the coding part of the chromosome cannot be changed. In the linear approximation all lengths $L=L$ (coding $)+L$ (telomere $)$ are allowed and if the corrections are small, L(telomere) is very sensitive to $L$ (stickyend).

The length of the sticky end rather than the length of the telomere would be the primary controller. The quite high strength of the longitudinal electric field is a surprise. An interesting prediction is that prokaryotes with circular DNA strands would have no wake-up-sleep cycle like eukaryotes. Viruses however have both circular and open strands.

(2) Minimization of the electrostatic energy taking into account interaction between nonnearest neighbors

What kind of corrections the inclusion of the Coulomb interactions of charges which are not nearest neighbors could bring in?

(a) Nearest neighbors have been identified as neighbors in transversal direction and it has been assumed that only DNA-DNA and DDNA-DDNA, and DNA-DDNA interactions matter. A better approximation would take into account the repulsive nearest-neighbor interactions between phosphates and between dark protons along dark DNA. Same applies to DNA-DDNA interactions.

(b) All these terms give a contribution proportional to $L$ and mean only a scaling of the parameter $n_{0}$, whose order of magnitude remains the same and by the presence of the longitudinal dipole electric field can be positive. 
(c) Consider the contribution of the interactions of given DNA codon and DDNA codon with the non-nearest neighbors along DNA and dark DNA. These interactions can be regarded as dipole and higher multiple interactions since the total charges of the codon pair DNA + DDNA vanish. In the lowest order approximation dipole-dipole interactions depending on the distance $r$ between dipoles like $1 / r^{3}$.

(d) Simple dimensional arguments give the general form of the dipole contributions. By dimensional considerations alone, the sum over dipole interaction energies for a given codon or nucleotide gives a contribution proportional to $1 / L^{2}$. Summing over these contributions gives a total contribution proportional to $1 / L$.

The dipole contribution is proportional to $(d n / d l)^{2}$, to the square of the dipole moments of a given nucleotide (codon). Since dipole moments are of the order $e R, R$ the transversal scale of DNA+DDNA system, individual dipole-dipole interaction energy is proportional to $e^{2} S$.

Therefore the Coulomb interaction energy would be of the general form

$$
E=\frac{e^{2} L}{S}\left[-n^{2}+n_{0}^{2}\right]+k e^{2}\left(\frac{d n}{d l}\right)^{2} \frac{S}{L}
$$

where $k$ is a numerical factor determined by the details of the model. Note that dark protons forming a dark variant of ordinary nucleus are expected to have also counterparts of strong interactions expected to be short ranged.

(e) The minimization of energy would give:

$$
\left.\left.F=-\frac{d E}{d L}=\frac{e^{2}}{S}\left[-n^{2}+n_{0}^{2}\right]-k e^{2}(d n / d l)^{2} \frac{S}{L^{2}}\right)\right]=0
$$

This gives for $L(n)$ :

$$
L(n)=\frac{d n}{d l} S \sqrt{\frac{k}{-n^{2}+n_{0}^{2}}}
$$

The condition that the argument of square root in non-negative, implies that one must have either $\left(k>0, n<n_{0}\right)$ or $\left(k<0, n>n_{0}\right) . n<n_{0}$ option seems to be the physical one.

(f) $n<n_{0}$ requires $k>0$ so that the dipole interaction energy is positive. For $\mathrm{n} \rightarrow 0$, $\mathrm{L}$ approaches to:

$$
L(0)=\frac{d n}{d l} S \sqrt{\frac{k}{n_{0}^{2}}}
$$

$L(0)$ could correspond to the length for the coding part of DNA (no telomere is allowed). At the limit $n \rightarrow \infty L(0)$ approaches infinite value and the length of the telomere becomes extremely sensitive to the value of $n$ and $n$ becomes an ideal control variable.

For $n>n_{0}$ one must have $k<0$ meaning that the contribution of the dipole-dipole interactions to the total energy is negative. The stable DNA length shortens roughly like $L \propto 1 / n$ as $n$ increases: this does not conform with the intuitive picture.

\subsection{Tests for the TGD based model of DNA as ferroelectret}

The standard physics view is that the possible ferroelectricity for DNA is due to the instantaneous polarization of codons A,T,C,G in external field which is proportional to electric field $E$ if the polarization vanishes for $E=0$. Ferroelectriy is analogous to spontaneous magnetism that there is electric field also for $E=0$ : this requires permanent electric dipole moments generated by small external field an left when the field is taken to zero.

In [31] a model for the polarizability of nucleotides A,T,C, G is developed based on standard physics so that the external electric field would generate dipole moment for given nucleotide. What one hopes to have is ferro-electric behavior. The model calculations give ferroelectric behavior and a square shaped hysteresis curve. In case of entire DNA each nucleotide would behave independently in inhomogenous electric field with varying direction.

Also in [19] the dipole moments are estimated for both bases and nu- cleotides, and the esimated dipole moments are in the range of 2-6 Debyes $(D=0.02 \mathrm{enm})$ that is $0.04-0.12 \mathrm{enm}$. TGD estimate for the electric field is about $n e / S, S=\pi R^{2}$ the effective area of the flux tube assignable to DNA + dark DNA.

The first thing to notice is that the flux would be along entire DNA, not only the telomere and the overhangs portions carry the charges creating the electric field along DNA. Electric flux flows along DNA. Telomere would be a kind of buffer against the evil world. Overhang/sticky ends could play a key role in control of the arrow of time for DNA. Similar mechanism would be at work at the level of entire body changing the direction of endogenous electric field and leading to wake-up to sleep or vice versa [5]. 
Suppose that the charges at the opposite ends of DNA are of opposite sign. An unnecessary strong assumption is that they are of the same magnitude. The dipole moment would be roughly given by the difference $\mathrm{Q}_{1}-\mathrm{Q}_{2}$ of the charges multiplied by the distance $L$ between ends of the chromosome along the DNA strand. Note that the channeling of electric flux along DNA would be rely on TGD view about space allowing monopole flux tubes whose deformations carry also electric field.

The static electric field would be realized as a conserved electric flux along the entire DNA, not only telomere. The order of magnitude is $10 \mathrm{GV} / \mathrm{m}$ for $R=1 \mathrm{~nm}$ so that it would be rather strong. The strength of electric field is proportional to $1 / R^{2}$ and $R$ is expected to vary in the range $1-10 \mathrm{~nm}$. Note that $L(151)=10 \mathrm{~nm}$ corresponds to the p-adic length scaled the thickness of the DNA coil and chromosome thickness.

The effective dipole moment per nucleotide would be $p \cong n e d \cong n \times 0.3$ enm and quantized as multiples of $n$. The estimate is at most by a factor 2.2-7.5 larger than the estimates from the atomic contributions and would allow selecting between the standard model and TGD based model.

\subsubsection{Nanoscopic implications}

What could be possible experimental consequences of the proposed electric field? Consider first the situation at the level of single DNA double strand.

(1) The accelerated motion of a test charge along DNA could serve as a test for this option One can consider both quantum motion without dissipation - perhaps along the dark DNA - and Ohmic current along the ordinary DNA. They would run also in absence of external electric field unlike ordinary Ohmic currents.

These currents could be nanoscopic analogs of the DC currents observed by Becker in body scale and brain scale. If they are steady currents the current is conserved and must return so that a closed current loop is formed. The currents could be also pulselike taking surplus dark protons between ends of the chromosomes and changing the their roles. This would be quantum event associated with BSFR and could mean time reversal.

Electronic (not protonic) currents along DNA [32] have been observed for single DNA strands in an external electric and it is found that the con- ductivity is surprisingly high. In the recent case conduction double strand property and sticky ends would be essential.

(2) How could the current return in steady situation? This question must be answered also for Becker's current. Does the current flow as Ohmic current along ordinary DNA and return back along the dark DNA as non-dissipative current? The proton current along DNA along electric field to negatively charged and dark protons would be accelerating: the quantum description would correspond to a particle in linear potential, which is standard quantum mechanical problem. The larger the charge (the length of the sticky end), the stronger the current. Its magnitude would be quantized being proportional to the length and charge ne of the sticky end. The variation of sticky end length would vary the strength of the current.

The larger the charge (the length of the sticky end), the stronger the current. Its magnitude would be quantized being proportional to the length and charge ne of the sticky end. The variation of sticky end length would vary the strength of the current. There is evidence for proton AC current conduction in the DNA double strandimidazole composite material under anhydrous conditions (no water) in the freqency range $4 \mathrm{~Hz}-1 \mathrm{MHz}$ [33]. If the mechanism is the proposed one - probably not - the oscillatory current could correspond to occurrence of BSFRs changing the arrow of time per each period of $T=1 / f$. This would predict the current to be $I=2 n e f$, where $\pm n e$ are the charges at the ends of the double DNA strand.

\subsubsection{How to test whether DNA double strand is ferroelectret?}

(1) The measurement of the possible longitudinal electric field of DNA and its correlation with the length of the telomere or of the sticky end would be an interesting expermental project. DNA exconuclease restriction enzyme allowing to cut pieces from the end of either DNA strand could allow creation of desired length of unpaired portion of DNA. Also blunt ends could be created and the prediction is that there is no electric field in this case.

(2) The telomere or the entire DNA would be like a dipole and would interact with external electric fields. One should be able to prepare a DNA sample as an electret so that DNAs would have the same dipole direction and this structure could be put in an electric field allowing measuring the dipole moment of DNA as a macroscopic motion in the field.

The external electric field would give rise to a torque acting on the entire DNA double strand. If nucleotides behave as independent dipoles as the standard physics based model suggests, 
this would not be the case and the dipole moments of the nucleotides would only turn in the direction of the external field.

(3) One could also study whether and how the possible DNA dipole moment is affected by the telomerase affecting the length of telomere. The first guess would be that is the length of the sticky end which is affected and that the length of the telomere correlates with this by stability conditions. Pyroelectricity and piezoelectricity and the use of external electric field produce ferroelectrets from various biological tissues $[25,26]$. These methods applied to DNA crystals [20] could allow to test the hypothesis.

The measurement of the possible longitudinal electric field of chromosome or DNA double strand and its correlation with its length could serve as an early bioelectric marker: this could be an experimental project. Currently, the measurement of telomere length by quantitative PCR is quite common and for a summary of critical factors and recommendations for assay design, interested readers may see [34]. Also, a full description and protocol for examination of the telomere G-overhang structure in different plant, human and vertebrate models are available [18,35-37]. However, to the best of our knowledge, this is the first hypothetical paper to suggest DNA/telomere's bioelectric field as an independent marker which may revolutionize a wide array of medical investigations.

\subsubsection{Could pyroelectricity, piezoelectricity, or the behavior in external electric fields be used to demonstrate that DNA has a longitudinal internal electric field}

One can consider also the consequences at condensed matter level. Athensteadt has found [38] that it is possible to make various tissues of vertebrates piezoelectric or pyroelectric.

Pyroelectric materials [38] are crystals in which the change of the temperature involving thermal energy flow induces a macroscopic electric polarization and therefore electric field making the material ferroelectric. In piezo-electric materials [38] mechanical stress induces a generation of polarization and macroscopic electric field. Also an external electric field can induce polarization producing a ferroelectret.

One can visualize the situation using a triangle having kinetic, electric, and thermal energies as corners. For piezoelectric materials the motion occurs along the edge connect- ing electric and mechanical energy. For pyroelectric materials the motion occurs along the edge connecting electric and thermal energy.

The proposal is that DNA double strand + dark DNA strand carries internal electric field is 1-D ferroelectric aperiodic crystal due to its inherent polarization. One cannot exclude the possibility that also single DNA strand + dark strand has this property. DNA should be in vivo state. DNA crystals [20] might allow to test the phenomenon. For instance, it is known that DNA suspended in liquid which is evaporated forms crystal [21]. Could DNA crystals become ferroelectrets by heating or cooling or by applying a mechanical stress or an external electric field?

If this would occur, the interpretation would be that DNA strands become parallel and have parallel electric fields giving rise to ferroelectricity. In the positive case, one could test the hypothesis by using DNA preparations with different values of $n$ for the number of overhang nucleotides: electric field in the ideal situation would be proportional to $n$ if the area density of the parallel DNA strands is the same.

\subsection{Medical application of the measurement of bioelectric fields of DNA}

Thought experiments can be designed in order to possibly carry out in a sample of volunteer identical twins with quite matched or similar lifestyle to find out any correlation between age and gender- adjusted death rates and cancer measures with differential and mean whole-body DNA bioelectric field, with minimally invasive techniques and data collection to derive a regression equation as a rough estimate. Severity and progression of cancer, time of death and DNA bioelectric field for each sibling can be recorded for analysis and data mining purposes and assessment of prognostic and predictive values.

We suggest that future clinical trials investigating telomere rejuvenation in aging and cancer studies, measure appropriate variables such as DNA bioelectric field, percent of apoptotic and cancer cells, severity and progression of cancer and such as those to yield preliminary data for future interventional trials and ethics committees. Interventional studies in animal models of cancer and aging using telomere rejuvenation techniques would be the first steps to find out any 
causal effect of restored DNA's bioelectric field -to level at birth- on therapeutic measures of cancers and aging.

It will be also useful to design a registry for DNA bioelectric field strengths in different cell types at birth (as baseline value), incidence of major cancers by type, and death, to find out prospectively a golden ratio at which highest incidence of cancers and death is recorded, after controlling for compounding factors. Such data in future can be used for telomere rejuvenation in a prophylactic manner, if needed. DNA bioelectric field measure could have some advances of the measurement of telomere length. Being less invasive and preserving the patients ability to undergo possible repeat operations, reasonable cost-effectiveness which allows multiple measurements and replications, lesser inter and intra-subject and observer variations compared to that of other discrete measures due to the continuous nature of electric field measures which additionally allows more reliable and valid comparisons of percentile-effects for DNA bioelectric field restorations or variations on measures of interest, just to mention a few among others.

To set up individualized cut-off values, differential and mean whole-body DNA bioferroelectric field at birth; and for normal range values, data obtained from different age and gender groups can be used as a preliminary start point, respectively.

\section{Conclusions}

Telomeres bioelectric field could serve as a novel bioelectric marker to be used for prognostic and diagnostic purposes in researches of cancer, aging, surgery grafts and rejuvenation.

\section{Competing interests}

The authors declare that they have no competing interests.

\section{Authors' contributions}

Matti Pitkänen wrote the initial draft, revised and finalized the paper. Reza Rastmanesh criticized the paper, revised and approved the paper.

\section{References}

[1] Athenstaedt H. Pyroelectric and piezoelectric properties of vertebrates. Annals of the New York Academy of Sciences, 1974, 238(1): 68-94 https://doi.org/10.1111/j.1749-6632.1974.tb26780.x

[2] Yam SC, Zain SM, Lee VS, et al. Correlation between polar surface area and bioferroelectricity in DNA and RNA nucleobases. The European Physical Journal E, 2018, 41(7): 86. https://doi.org/10.1140/epje/i2018-11696-5

[3] Pitkänen M. Zero Energy Ontology \& Consciousness. Journal of Consciousness Exploration \& Research, 2020, 11(1): 1-9.

[4] Pitkaanen M. Topological Geometrodynamics: Revised Edition. Bentham, 2016. https://doi.org/10.2174/97816810817931160101

[5] Becker RO and Selden G. The Body Electric: Electromagnetism and the Foundation of Life. William Morrow \& Company, Inc. , New York, 1990.

[6] Ma H, Zhou Z, Wei S, et al. Shortened telomere length is associated with increased risk of cancer: a meta-analysis. PloS one, 2011, 6(6): e20466. https://doi.org/10.1371/journal.pone.0020466

[7] Haycock PC, Burgess S, Nounu A, et al. Association between telomere length and risk of cancer and non-neoplastic diseases: a Mendelian randomization study. JAMA oncology, 2017, 3(5): 636-651. https://doi.org/10.1001/jamaoncol.2016.5945

[8] Barthel FP, Wei W, Tang M, et al. Systematic analysis of telomere length and somatic alterations in 31 cancer types. Nature genetics, 2017, 49(3): 349-357. https://doi.org/10.1038/ng.3781

[9] Autexier C and Greider CW. Telomerase and cancer: revisiting the telomere hypothesis. Trends in biochemical sciences, 1996, 21(10): 387-391. https://doi.org/10.1016/S0968-0004(96)10042-6

[10] Shay JW, Zou Y, Hiyama E, et al. Telomerase and cancer. Human molecular genetics, 2001, 10(7): 677-685.

https://doi.org/10.1093/hmg/10.7.677 
[11] Zheng Y, Zhang F, Sun B, et al. Telomerase enzymatic component hTERT shortens long telomeres in human cells. Cell cycle (Georgetown, Tex.), 2014, 13(11): 1765-1776. https://doi.org/10.4161/cc.28705

[12] Rashid-Kolvear F, Pintiliet M and Done SJ. Telomere Length on Chromosome 17q Shortens More than Global Telomere Length in the Development of Breast Cancer. Neoplasia (New York, N.Y.), 2007, 9(4): 265-270. https://doi.org/10.1593/neo.07106

[13] Chai W, Shay JW and Wright WE. Human Telomeres Maintain Their Overhang Length at Senescence. Molecular \& Cellular Biology, 2005, 25(6): 2158-2168. https://doi.org/10.1128/MCB.25.6.2158-2168.2005

[14] Hiyama E and Hiyama K. Telomere and telomerase in stem cells. British Journal of Cancer, 2007, 96(7): 1020-1024. https://doi.org/10.1038/sj.bjc.6603671

[15] Guerrant RL. Evolution of Evolution: The Survival Value of Caring Cambridge Scholars Publishing, 2018.

[16] Wikipedia, Telomere, 2020.

[17] Wikipedia, Sticky and Blunt Ends, 2020.

[18] Sandhu R and Li B. Examination of the telomere G-overhang structure in Trypanosoma brucei. Journal of Visualized Experiments, 2011, 47: e1959. https://doi.org/10.3791/1959

[19] Berezhnoy AY and Duplij SA. Dependence of nucleotide physical properties on their placement in codons and determinative degree. Journal of Zhejiang University SCIENCE B, 2005, 6(10): 948-960. https://doi.org/10.1631/jzus.2005.B0948

[20] Seeman NC. An overview of structural DNA nanotechnology. Molecular biotechnology, 2007, 37(3): 246. https://doi.org/10.1007/s12033-007-0059-4

[21] Gledhil L. Turns out crystallized DNA is crazy pretty, 2020.

[22] Pokorny J, Jelinek F, Trkal V, et al. Vibrations in microtubules. Journal of Biological Physics, 1997, 23(3): 171-179. https://doi.org/10.1023/A:1005092601078

[23] Pokorn J, Jelnek F and Trkal V. Electric field around microtubules. Bioelectrochemistry and Bioenergetics, 1998, 45(2): 239-245. https://doi.org/10.1016/S0302-4598(98)00100-7

[24] Wang J, Eisenstatt JR, Audry J, et al. A Heterochromatin Domain Forms Gradually at a New Telomere and Is Dynamic at Stable Telomeres. Molecular \& Cellular Biology, 2018, 38(15): MCB.00393-17. https://doi.org/10.1128/MCB.00393-17

[25] Wikipedia, Chromosome, 2020.

[26] Wikipedia, Ferroelectricity, 2020.

[27] Pitkänen M. Getting philosophical: some comments about the problems of physics, neuroscience, and biology. DNA Decipher Journal, 2018. https://doi.org/10.13140/RG.2.2.19239.91048

[28] Pitkänen M. Philosophy of Adelic Physics, in New Trends and Advanced Methods in Interdisciplinary Mathematical Sciences Springer, 2017: 241-319. https://doi.org/10.1007/978-3-319-55612-3_11

[29] Pitkänen M. On the Correspondence of Dark Nuclear Genetic Code \& Ordinary Genetic Code, Scientific GOD Journal, 2018.

[30] Pitkänen M. An Overall View about Models of Genetic Code \& Biopharmony. DNA Decipher Journal, 2019.

[31] See-Chuan Y, Md. ZS, Vannajan SL, et al. Correlation between polar surface area and bioferroelectricity in DNA and RNA nucleobases. European Physical Journal E, 2018, 41(7): 86. https://doi.org/10.1140/epje/i2018-11696-5

[32] Dekker C and Ratner M. Electronic properties of DNA. Physics World, 2001, 14(8): 29. https://doi.org/10.1088/2058-7058/14/8/33

[33] Yamada M and Goto A. Proton conduction of DNA-imidazole composite material under anhydrous condition. Polymer journal, 2012, 44(5): 415-420, . https://doi.org/10.1038/pj.2012.5

[34] Lin J, Smith DL, Esteves K, et al. Telomere length measurement by qPCR-Summary of critical factors and recommendations for assay design. Psychoneuroendocrinology, 2019, 99: 271-278. https://doi.org/10.1016/j.psyneuen.2018.10.005

[35] Dai X, Huang C, Bhusari A, et al. Molecular steps of G-overhang generation at human telomeres and its function in chromosome end protection. EMBO Journal, 2010, 29(16): 2788-2801. https://doi.org/10.1038/emboj.2010.156

[36] Hansel R, Lohr F, Trantirek L, et al. High-resolution insight into G-overhang architecture. Journal of the American Chemical Society, 2013, 135(7): 2816-2824. https://doi.org/10.1021/ja312403b 
[37] Riha K, Mcknight TD, Fajkus J, et al. Analysis of the G-overhang structures on plant telomeres: evidence for two distinct telomere architectures. Plant Journal for Cell \& Molecular Biology, 2010, 23(5): 633-641.

https://doi.org/10.1046/j.1365-313x.2000.00831.x

[38] Athenstaedt H. Pyroelectric and piezoelectric properties of vertebrates. Annals of the New York Academy of Sciences, 1974, 238: 68-94.

https://doi.org/10.1111/j.1749-6632.1974.tb26780.x

(Edited by Snowy Wang) 